Nils Reiter, Gerhard Kremer, Kerstin Jung, Benjamin Krautter, Janis Pagel und Axel Pichler

\title{
Reaching out: Interdisziplinäre Kommunikation und Dissemination
}

\author{
Ein CRETA-Erfahrungsbericht
}

Zusammenfassung: In diesem Kapitel diskutieren wir Aktivitäten, die wir im Rahmen von CRETA etabliert haben, um einerseits die interdisziplinäre Kommunikation zu verbessern und andererseits CRETA-Erkenntnisse nach außen zu tragen. Konkret stellen wir ein mehrfach durchgeführtes hackatorial (Workshop „Maschinelles Lernen lernen“), einen Workshop zur Operationalisierung als Kernaufgabe für die Digital Humanities, sowie das CRETA-Coaching vor. Zu allen Aktivitäten sammeln wir unsere Ergebnisse und Erfahrungen in einem Fazit.

Abstract: This chapter presents various activities related to internal and external communication, including activities related to the dissemination of ideas developed in CRETA. Specifically, we present the 'hackatorial' (workshop "Learning machine learning”), a 'workshop on operationalization' as a core task for the digital humanities, and the 'CRETA coaching'. For all activities we collect our results and experiences in a conclusion.

\section{Einleitung}

Neben die inhaltlichen und fachlichen Herausforderungen treten im Bereich der Digital Humanities die, die sich aus der Interdisziplinarität der Projektteams ergeben. Da es sich auch bei CRETA um ein interdisziplinär zusammengesetztes Zentrum handelt, waren die Themen Kommunikation und Weiterbildung von Anfang an präsent. ${ }^{1}$ Eine funktionierende interdisziplinäre Kommunikation ist dabei eine der größten Herausforderungen. Suditsch (2017) zufolge besteht das Problem im Kern darin, dass in interdisziplinären Projekten zwar Expertinnen und Experten

1 Aus diesem Grund findet sich auch eine stilisierte Sprechblase im CRETA-Logo: 0 .

Nils Reiter, Gerhard Kremer, Kerstin Jung, Janis Pagel, Institut für Maschinelle Sprachverarbeitung, Universität Stuttgart

Axel Pichler, Stuttgart Research Center for Text Studies, Universität Stuttgart

Benjamin Krautter, Germanistisches Seminar, Universität Heidelberg

¿ Open Access. ( 2020 Nils Reiter, Gerhard Kremer, Kerstin Jung, Benjamin Krautter, Janis Pagel und Axel Pichler; publiziert von De Gruyter (c) BY-NC-ND Dieses Werk ist lizenziert unter der Creative Commons AttributionNonCommercial-NoDerivatives 4.0 International Lizenz. https://doi.org/10.1515/9783110693973-019 
zusammenarbeiten, diese aber in unterschiedlichen Fächern Expertinnen und Experten sind. Gerade die ,[Elemente] (z. B. gemeinsamer Wissenshintergrund, gemeinsame Fachsprache), die innerhalb einer Disziplin als gesetzt gelten, müssen in interdisziplinärer Zusammenarbeit neu ausdiskutiert werden“ (Suditsch 2017, S. 2). Dies führt zum sogenannten Fachsprachen-Paradox:

Fachsprachen sind paradox, da sie innerhalb von Fachgemeinschaften explizite und effiziente Verständigung ermöglichen, während sie interdisziplinäre Kommunikation anfällig für Missverständnisse machen und ineffizient sind. (Suditsch 2017, S. 41)

Fachsprachen sind deswegen anfällig für Missverständnisse, weil Ausdrücke oft mehrfach belegt sind: Mit dem Wort ,Modell` $z$. B. verbinden sowohl Forschende aus der Computerlinguistik, Literaturwissenschaft und Philosophie etwas - aber nicht das gleiche. ${ }^{2}$ Wenn ein Wort je nach Disziplin verschiedene Bedeutungen annimmt, ist das meistens nicht von Anfang an offensichtlich, sondern muss erst freigelegt werden. Dieses Freilegen ist ein mühsamer Prozess, da Fachsprachen von ihren Anwendenden stark internalisiert sind und wenig bewusst verwendet werden. Letzten Endes braucht es in solchen Projekten zumindest ein paar Mitarbeitende, die die jeweils andere Disziplin zu verstehen versuchen, und immer wieder nachbohren. Für die Verständigung auf gemeinsame Begriffe und ihre Bedeutung (,Entwicklung einer gemeinsamen Sprache') ist der informelle Austausch von großer Wichtigkeit: „Interdisziplinäre Projekte sind für die meisten Beteiligten immer noch eine neue Erfahrung; die daraus entstehenden Unsicherheiten lassen sich auf informeller Ebene am schnellsten ausräumen“ (Lengwiler 2005, S. 54).

In CRETA werden diese Herausforderungen auf mehreren Ebenen adressiert. In regelmäßigem, bisher halbjährlichem Abstand treffen sich alle an CRETA Beteiligten zur sog. CRETA-Werkstatt. Bei diesen zwei- bis dreitägigen Veranstaltungen wird einerseits in Vorträgen aus aktuellen Arbeiten der Mitarbeitenden von CRETA berichtet. Andererseits wird das Treffen von der Arbeit in Kleingruppen bereichert, das etwa die andere Hälfte der Zeit einnimmt. Thema und Format der Gruppenarbeit können dabei sehr unterschiedlich sein. So kann es Gruppen geben, die an konkreten Annotationsaufgaben (oder -richtlinien) arbeiten, während andere aktuelle Forschungsthemen oder -publikationen aus ihrem Bereich diskutieren und wieder andere die nächsten Arbeitsschritte in ihren Projekten planen. Der Vorteil der Arbeit in Kleingruppen liegt nicht nur darin, dass so im Rahmen der Werkstatt häufig inhaltliche Ergebnisse produziert werden, son-

2 Siehe hierzu auch den Abschnitt „Hintergrund: Modelle und Modellierung in der algorithmischen Textanalyse“ in der Einleitung des Bandes auf Seite 12. 
dern auch darin, dass sich auch diejenigen CRETA-Beteiligten aktiv in die CRETAWerkstatt einbringen können, die keinen Vortrag halten. Der kleinere Rahmen einer Arbeitsgruppe macht es auch leichter, Unsicherheiten und Unklarheiten auszuräumen. Es wird dabei auf die Zugänglichkeit und Offenheit für den wissenschaftlichen Nachwuchs geachtet sowie auf eine möglichst diverse Zusammensetzung der Arbeitsgruppen (in Bezug auf Disziplinen, Hierarchiestufen und Geschlechter). Nicht zuletzt weil die CRETA-Beteiligten in Stuttgart über mehrere Standorte verteilt sind, fand auch - zusätzlich zu den Werkstätten - ein regelmäBiger Stammtisch statt, bei dem ein informeller Austausch möglich war.

Als projektinterne Weiterbildungen wurden zunächst einige Themen von CRETA-Beteiligten an andere CRETA-Beteiligte vermittelt (z.B. Versionsverwaltung mit git, Was ist eigentlich Hermeneutik?, Einführung in die Statistik, Maschinelles Lernen lernen, ...). Den Lehrenden führten diese Aktivitäten regelmäßig vor Augen, welche Vorannahmen sie implizit treffen, die im interdisziplinären Kontext neu ausgehandelt werden müssen. Für die Lernenden bot sich die Gelegenheit, eben jene Vorannahmen aus ihnen fremden Disziplinen besser zu verstehen, und so auch fremd-disziplinäre Forschungspraktiken und deren Resultate besser einordnen zu können. Aufgrund der positiven Erfahrungen dieser Formate wurden sie dann ausgeweitet und auch CRETA-extern im Rahmen von Workshops und Tutorials angeboten.

In diesem Kapitel stellen wir eine Auswahl dieser über die Jahre weiterentwickelten Aktivitäten dar (Tabelle 1 zeigt eine Übersicht). In Abschnitt 2 präsentieren wir das von uns entwickelte ,hackatorial'-Konzept, das wir CRETA-intern sowie im Rahmen mehrerer Digital-Humanities-Konferenzen erfolgreich durchgeführt haben. Ziel dabei war es, die Teilnehmenden mit Grundlagen maschineller Lernverfahren vertraut $\mathrm{zu}$ machen und ihnen ein know how zu vermitteln, mit Hilfe dessen sie a) sich auf informierte Weise den eigenen Aufgaben widmen und b) Ergebnisse maschineller Lernverfahren besser einschätzen können. In Abschnitt 3 besprechen wir ein darauf aufbauendes, ergänzendes Workshop-Konzept, das im Jahr 2020 zum ersten Mal durchgeführt wurde. Der Fokus lag dabei auf der Operationalisierung geisteswissenschaftlicher Forschungsfragen und den verschiedenen Möglichkeiten, sich diesem Problem zu nähern. Während die beiden Workshops sich an ein Publikum von 20 bis 35 Teilnehmenden richteten, haben wir 2019 auch ein stark individualisiertes Coaching-Programm angeboten, an dem vier Nachwuchswissenschaftlerinnen teilgenommen haben (Abschnitt 4). Dabei haben wir unsere - auch in diesem Buch gesammelte - Kompetenz bei der Operationalisierung gemeinsam mit den zu Coachenden auf neue Fragen bzw. Probleme angewendet, um ihnen konkrete Handlungsempfehlungen zu geben. Neben der praktischen Wissenserweiterung der Teilnehmenden an unseren Disseminationsprojekten konnte bei deren Entwicklung und Durchführung wiederum das 
Tab. 1: Tutorials und Workshops zu CRETA-Themen. DH: Jahrestagung der Alliance of Digital Humanities Organizations, DHd: Jahrestagung des Verbandes der Digital Humanities im deutschsprachigen Raum, ESU: European Summer University in Digital Humanities, LSS-ML: Late Summer School on Machine Learning for Language Analysis, HCH: Heidelberg Computational Humanities School.

\begin{tabular}{|c|c|c|c|}
\hline Datum & Ort & Titel & Verantwortliche \\
\hline 08.08 .2017 & DH, Montreal & $\begin{array}{l}\text { From Texts to Networks: } \\
\text { Combining Entity and } \\
\text { Segment Annotations in the } \\
\text { Analysis of Large Text } \\
\text { Corpora }\end{array}$ & $\begin{array}{l}\text { Nils Reiter, } \\
\text { Maximilian Overbeck, } \\
\text { Sandra Murr }\end{array}$ \\
\hline 26.02.2018 & DHd, Köln & $\begin{array}{l}\text { Maschinelles Lernen lernen: } \\
\text { Ein CRETA-Hackatorial zur } \\
\text { reflektierten automatischen } \\
\text { Textanalyse }\end{array}$ & $\begin{array}{l}\text { Nils Reiter, Nora } \\
\text { Ketschik, Gerhard } \\
\text { Kremer, Sarah } \\
\text { Schulz }\end{array}$ \\
\hline 23. -27.07 .2018 & ESU, Leipzig & $\begin{array}{l}\text { Reflektierte Textanalyse in } \\
\text { den Digital Humanities }\end{array}$ & $\begin{array}{l}\text { Nils Reiter, Sarah } \\
\text { Schulz }\end{array}$ \\
\hline 26. -27.09 .2018 & LSS-ML, Köln & Learning Machine Learning & Nils Reiter \\
\hline 26.03.2019 & DHd, Mainz & $\begin{array}{l}\text { Maschinelles Lernen lernen: } \\
\text { Ein CRETA-Hackatorial zur } \\
\text { reflektierten automatischen } \\
\text { Textanalyse }\end{array}$ & $\begin{array}{l}\text { Gerhard Kremer, } \\
\text { Kerstin Jung }\end{array}$ \\
\hline 15.07.2019 & $\mathrm{HCH}$, Heidelberg & $\begin{array}{l}\text { Quantitative Drama } \\
\text { Analytics }\end{array}$ & $\begin{array}{l}\text { Benjamin Krautter, } \\
\text { Janis Pagel, Nils } \\
\text { Reiter, Marcus } \\
\text { Willand }\end{array}$ \\
\hline 09.-17.09.2019 & Stuttgart & CRETA-Coaching & $\begin{array}{l}\text { Nils Reiter, Axel } \\
\text { Pichler }\end{array}$ \\
\hline 09.-20.09.2019 & Stuttgart & $\begin{array}{l}\text { Herbstschule der Sektion } \\
\text { Computerlinguistik der } \\
\text { Deutschen Gesellschaft für } \\
\text { Sprachwissenschaft }\end{array}$ & $\begin{array}{l}\text { Sebastian Padó, } \\
\text { Gerhard Kremer, } \\
\text { Jonas Kuhn, Sybille } \\
\text { Laderer, Nils Reiter, } \\
\text { Sabine Schulte im } \\
\text { Walde }\end{array}$ \\
\hline WS $2019 / 20$ & TU Darmstadt & $\begin{array}{l}\text { Seminar Deep Learning \& } \\
\text { Digital Humanities }\end{array}$ & $\begin{array}{l}\text { Steffen Eger, Thomas } \\
\text { Haider }\end{array}$ \\
\hline 03.03 .2020 & DHd, Paderborn & $\begin{array}{l}\text { Maschinelles Lernen lernen: } \\
\text { Ein CRETA-Hackatorial zur } \\
\text { reflektierten automatischen } \\
\text { Textanalyse }\end{array}$ & $\begin{array}{l}\text { Gerhard Kremer, } \\
\text { Kerstin Jung }\end{array}$ \\
\hline 03.03 .2020 & DHd, Paderborn & $\begin{array}{l}\text { Vom Phänomen zur Analyse. } \\
\text { Ein CRETA-Workshop zur } \\
\text { reflektierten } \\
\text { Operationalisierung in den } \\
\text { DH }\end{array}$ & $\begin{array}{l}\text { Nora Ketschik, } \\
\text { Benjamin Krautter, } \\
\text { Sandra Murr, Janis } \\
\text { Pagel, Nils Reiter }\end{array}$ \\
\hline
\end{tabular}


CRETA-Team Erfahrungen sammeln, die zur Verfeinerung der eigenen Methodenarbeit beigetragen haben.

\section{Hackatorial: Maschinelles Lernen lernen}

\subsection{Einleitung}

Ein mehrmals durchgeführter, halbtägiger Workshop (,Maschinelles Lernen lernen: Ein CRETA-Hackatorial zur reflektierten automatischen Textanalyse“) machte Teilnehmende praxisbezogen mit dem Thema des maschinellen Lernens vertraut. Der Titel nutzt die Kurzform hackatorial, angelehnt an sogenannte hackathons (aus engl. to hack und marathon), bei denen große Gruppen in einem kurzen Zeitrahmen Softwareentwicklung betreiben. Unser Ansatz bei „Maschinelles Lernen lernen“ ist es, dieses Prinzip mit einem Tutorial, also einer Anleitung zu verbinden.

Ziel dieses hackatorials war, den Teilnehmenden konkrete und praktische Einblicke in einen Standardfall automatischer Textanalyse zu geben. Am Beispiel der automatischen Erkennung von Entitätenreferenzen (siehe hierzu auch den Beitrag von Ketschik, Blessing et al. (2020), ab Seite 204 in diesem Band) ging das CRETA-Team auf allgemeine Annahmen, Verfahrensweisen und methodische Standards bei maschinellen Lernverfahren ein. Die Teilnehmenden konnten beim Bearbeiten von lauffähigem Programmiercode den Entscheidungsraum solcher Verfahren ausleuchten und bekamen einen zusammenhängenden Überblick von der manuellen Annotation ausgewählter Texte über die Feinjustierung der Lernverfahren bis zur Evaluation der Ergebnisse. Es wurden dabei keinerlei Vorkenntnisse über maschinelles Lernen oder Programmierkenntnisse vorausgesetzt.

Neben einem Python-Programm für das automatische Annotieren von Entitätenreferenzen, mit und an dem während des Tutorials gearbeitet wurde, stellten wir ein heterogenes, manuell annotiertes Korpus (bestehend aus verschiedenen, separaten Teilkorpora) sowie Routinen zur Evaluation und zum Vergleich von Annotationen zu Verfügung. Das Korpus enthält Entitätenreferenzen, die in CRETA annotiert wurden und deckt Texte verschiedener Disziplinen und Sprachstufen $\mathrm{ab}$.

Durch Einblick in die technische Umsetzung bekamen die Teilnehmenden ein Verständnis für die Grenzen und Möglichkeiten der Automatisierung, das sie dazu befähigt, zum einen das Potenzial solcher Verfahren für eigene Vorhaben realistisch(er) einzuschätzen, zum anderen aber auch Ergebnisse, die auf Basis 
Tab. 2: Korpora die im hackatorial verwendet wurden. Detaillierte Beschreibungen zu den Kategorien, Korpora und Annotationen können in Ketschik, Blessing et al. 2020 nachgeschlagen werden.

\begin{tabular}{llcc}
\hline Korpus & Sprache & Tokens & Entitätenreferenzen \\
\hline Werther & Neuhochdeutsch & 41505 & 331 \\
Parzival & Mittelhochdeutsch & 30491 & 2001 \\
Bundestagsdebatten & Neuhochdeutsch & 6371 & 488 \\
\hline
\end{tabular}

solcher Verfahren erzielt wurden, angemessen hinterfragen und interpretieren zu können.

\subsection{Entitätenreferenzen und Korpora}

Als Ausgangspunkt des Hackatorials diente uns das Konzept der Entität und ihrer Referenz, das in Ketschik, Blessing et al. 2020, S. 205 ff., ausgeführt und hier nur kurz wiederholt wird. Es wurde im Rahmen von CRETA bewusst weit gefasst und damit anschlussfähig für verschiedene Forschungsfragen aus den geistes- und sozialwissenschaftlichen Disziplinen. Insgesamt wurden fünf verschiedene Entitätenklassen bestimmt: PER (Personen/Figuren), LOC (Orte), ORG (Organisationen), abstrakte Konzepte (CNCs) und Werke (WRK). ,Entität' steht folglich für ein reales, fiktives oder mögliches Objekt, auf das von der Textoberfläche mittels eines einzelnen Wortes oder einer abgegrenzten Wortfolge referiert werden kann. Unter Entitätenreferenzen verstehen wir Ausdrücke, die auf eine Entität in der realen oder fiktiven Welt referieren. Das sind zum einen Eigennamen (named entities, z. B. „Peter“), zum anderen Gattungsnamen (z. B. „der Bauer“), sofern diese sich auf eine konkrete Instanz der Gattung beziehen. In CRETA wurden vier Korpora mit Entitätenreferenzen annotiert, von denen drei im hackatorial Verwendung fanden (Tabelle 2 zeigt eine Übersicht über die Charakteristika der Korpora).

\subsection{Ablauf}

Der Ablauf des Tutorials orientierte sich an sog. shared tasks aus der Computerlinguistik (vgl. Willand et al. 2020, S. 396f.), wobei der Aspekt des Wettbewerbs im Tutorial vor allem spielerischen Charakter hatte. Bei einem traditionellen shared task arbeiten die teilnehmenden Teams, oft auf Basis gleicher Daten, an Lösungen für eine einzelne gestellte Aufgabe. Solch eine definierte Aufgabe kann z. B. partof-speech-tagging, also das automatische Erkennen von Wortarten, sein. Durch ei- 
ne zeitgleiche Evaluation auf demselben Goldstandard können die entwickelten Systeme direkt verglichen werden. In unserem Tutorial setzten wir dieses Konzept live und vor Ort um.

Die Teilnehmenden versuchten nach einer Einführung in die Annotationen und die Annotationspraxis, selbständig und unabhängig voneinander die optimale Kombination aus (a) maschinellem Lernverfahren und (b) Text- und WortMerkmalen (z. B. Groß- und Kleinschreibung oder Wortlänge) für das Training eines Modells zur Erkennung von Enitätenreferenzen zu wählen. Dabei war das Ziel, auf einem für die Teilnehmenden neuen Datensatz zu den Ergebnissen zu kommen, die dem Goldstandard der manuellen Annotation am ähnlichsten waren.

Das bedeutet, dass der Einfluss von berücksichtigten Merkmalen auf das Erkennen von Entitätenreferenzen empirisch getestet werden konnte. Dabei waren Intuitionen über die Daten und das annotierte Phänomen hilfreich, da simplem Durchprobieren aller möglichen Kombinationen zeitliche Grenzen gesetzt waren. Zusätzlich wurden bei jedem Testlauf Informationen über die Entscheidungen protokolliert, um die Erklärbarkeit der Ergebnisse zu unterstützen.

Wir verzichteten bewusst auf eine grafische Benutzerschnittstelle, um den Teilnehmenden den ,Blick hinter den Vorhang' zu ermöglichen. Viele grafische Benutzeroberflächen verstecken die Komplexität der beim machine learning nötigen Entscheidungsprozesse (vgl. Reiter, Kuhn et al. 2017). Ziel des hackatorial war es ja, gerade diese sichtbar zu machen. Die Teilnehmenden bearbeiteten das (Python-)Programm also nach einer Einführung und unter Aufsicht direkt in einem Texteditor. Vorkenntnisse in Python waren dabei nicht nötig: Das von uns zur Verfügung gestellte Programm war so aufgebaut, dass auch Neulinge relativ schnell die $\mathrm{zu}$ bearbeitenden Teile verstehen und damit experimentieren konnten, indem Features zu- und abgeschaltet werden konnten (im einfachsten Fall durch das Setzen von Kommentarzeichen). Teilnehmende mit Erfahrungen im Programmieren konnten komplexere Merkmale verwenden, indem sie eigene Python-Funktionen implementierten.

\section{Workshop: Operationalisierung in den Digital Humanities}

\subsection{Einleitung}

Spätestens seit Franco Morettis 2013 veröffentlichtem Literary-Lab-Pamphlet ,Operationalizing': or, the Function of Measurement in Modern Literary Theory (Moretti 2013) ist die Operationalisierung geisteswissenschaftlicher Kategorien und 
Konzepte zentrales Thema im Bereich der Digital Humanities (cf. Jacke 2014; Jannidis et al. 2016; Gius 2019; Pichler und Reiter 2020). Mit Moretti gesprochen gilt es, eine Brücke zu schlagen, und zwar von „concepts to measurement, and then to the world“ (Moretti 2013, S. 1). Der auf der DHd 2020 erstmalig durchgeführte Workshop adressierte diese spezifische Herausforderung der Messbarmachung theoretischer Konzepte anhand von drei Anwendungsbeispielen, die literaturund sozialwissenschaftliche Phänomene umfassten.

Ziel des Workshops war es einerseits, die Schnittstelle zwischen computergestützten Methoden und geisteswissenschaftlichen Kategorien als wichtigen Teil der Forschungsarbeit herauszustreichen. Dadurch sollte ein Bewusstsein für die disziplinären Unterschiede und Herausforderungen ausgebildet werden, die es bei der Verschränkung von Informationstechnik und Geisteswissenschaften zu überwinden gilt. Andererseits wurden typische Problemstellungen auch in der tatsächlichen Arbeitspraxis adressiert und Lösungsmöglichkeiten aufgezeigt. Dazu nutzten wir drei im Rahmen von CRETA umfangreich bearbeitete und beforschte Anwendungsszenarien: die Extraktion von Entitäten und Entitätenreferenzen (Ketschik, Blessing et al. 2020), die Segmentierung von Erzähltexten in verschiedene Erzählebenen (Barth 2020; Ketschik, Murr et al. 2020; Willand et al. 2020), sowie das holistische Textphänomen der sogenannten ,Wertherness‘ (Richter 2020). ${ }^{3}$ Die theoretische und praktische Auseinandersetzung mit diesen Phänomenen hatte zum Ziel, die einzelnen Schritte auf dem Weg zu einer angemessenen und reflektierten Operationalisierung geisteswissenschaftlicher Konzepte aufzuzeigen und mit Blick auf die Fragestellung und zu erwartenden Ergebnisse immer wieder kritisch zu hinterfragen. Denn nur eine durchdachte Operationalisierung erlaubt im Anschluss an die quantitative Auswertung eine adäquate und auch für die Fachdisziplinen relevante Interpretation der Ergebnisse.

\subsection{Verschiedene Ansätze für verschiedene Phänomene}

Die von uns gewählten Anwendungsszenarien waren als ,Prototypen-Repertoire gedacht, das verschiedene Verfahrensweisen der Operationalisierung vorstellt, diskutiert und reflektiert, zugleich aber auch Möglichkeiten der Übertragung auf andere Anwendungsfälle exemplarisch aufzeigt. Die Phänomene und ihre damit zusammenhängenden Analyseeinheiten sind in Tabelle 3 zusammengefasst.

3 Alle hier referenzierten Arbeiten sind Teile des vorliegenden Bandes. 
Tab. 3: Phänomene, die beim Workshop zur Operationalisierung verwendet wurden

\begin{tabular}{lll}
\hline Phänomen & Einheit & Datenquelle/Referenz \\
\hline Entitätenreferenzen & Wörter/Wortgruppen & Ketschik, Blessing et al. (2020) \\
Erzählebenen & Textsegmente & $\begin{array}{l}\text { Teil IV: Ketschik, Murr et al. (2020) und Willand } \\
\text { et al. (2020) }\end{array}$ \\
Wertherness & Ganze Texte & Richter (2020) \\
\hline
\end{tabular}

Der Workshop konzentrierte sich auf zwei verschiedene Ansätze der Operationalisierung, die sich - abhängig von Forschungsfrage und -phase - gegenseitig ergänzen können. ${ }^{4}$ Der erste Ansatz stellt die Definition von Konzepten oder Kategorien mittels Annotationen in den Mittelpunkt. (Manuelle) Annotationen dienen hierbei zur Schärfung der untersuchten Konzepte. Probleme, Unklarheiten oder nicht beachtete Teilphänomene, die der Annotationsprozess aufdecken kann, werden in die Definition zurückgespielt, die Konzepte und Kategorien geschärft. Dies hilft einerseits eine größere intersubjektive Übereinstimmung der Annotationen zu erzielen, kann aber auch in die Theoriediskussion einfließen und diese bereichern (vgl. Gius und Jacke 2017 oder Pagel et al. 2020, ab Seite 125 in diesem Band). Die intensive Auseinandersetzung mit dem Material und den annotierten Instanzen gibt zudem Impulse für die computergestützte Operationalisierung.

Der zweite Ansatz schlägt eine indirekte Operationalisierung der betrachteten Phänomene vor. Dabei werden mehrere messbare Eigenschaften betrachtet, die mit dem zu operationalisierenden Konzept zwar verwandt sind, es aber jeweils einzeln nicht vollständig abdecken (vgl. Sack 2011; Reiter und Willand 2018). Bei dieser indirekten Annäherung ist vor allem die Gesamtschau der verschiedenen Einflussfaktoren aufschlussreich. Dabei lassen sich strukturelle, holistische und linguistische Eigenschaften von Texten zusammenführen.

Wie schon im hackatorial verwendeten wir die in CRETA annotierten Entitätenreferenzen in mittelhochdeutschen Artusromane und Bundestagsdebatten (s. o.). Sie dienten hier als Beispiel für die Operationalisierung von lokalen Textphänomenen.

Als zweites Phänomen beschäftigten wir uns mit der narratologischen Bestimmung von Erzählebenen, die Erzähltexte seriell oder ineinander verschachtelt segmentieren. Erzählebenen stehen im Zentrum des in Teil IV beschriebenen shared tasks, (Willand et al. 2020, ab S. 391 in diesem Band). Grundlage der im

4 Die Ansätze spiegeln die beiden Pfade des von Pichler und Reiter (2020) ab Seite 43 in diesem Band diskutierten Workflows. 
Workshop verwendeten Daten waren die Richtlinien, die in Ketschik, Murr et al. 2020 ab S. 391 in diesem Band beschrieben werden. Im Gegensatz zu Entitätenreferenzen handelt es sich um ein stark variierendes Phänomen - eine Erzählebene kann eine einzelne Äußerung einer Figur umfassen, oder den Gutteil eines Textes. Außerdem können Erzählebenen in andere Erzählebenen eingebettet werden, sie sind also ggf. verschachtelt.

Das letzte Anwendungsbeispiel rückte ein holistisches Textphänomen, die sogenannte ,Wertherness', ins Zentrum der Überlegungen. Die ,Wertherness‘ bezeichnet eine Menge an Texteigenschaften, die Texte im Anschluss an Goethes 1774 veröffentlichten Briefroman Die Leiden des jungen Werthers als literarische Adaptationen, also ,Wertheriaden', identifizieren. Ursächlich dafür sind verschiedene Bezugnahmen auf den Ursprungstext, die formaler (etwa Briefroman) wie inhaltlicher (etwa Rolle der Natur, Verhältnis Subjekt-Gesellschaft, Dreiecksbeziehung) Natur sein können. Für eine computergestützte Analyse der ,Wertheriaden' bietet sich hier eine indirekte Operationalisierung an - welche messbaren Eigenschaften deuten auf eine Wertheriade hin? Welche der Texteigenschaften sind messbar? Neben der Identifikation der verschiedenen Eigenschaften, gilt es zusätzlich, ihre Kombination in bekannten ,Wertheriaden“ auszuloten.

\subsection{Ablauf des Workshops}

Der Ablauf des Workshops teilte sich in Theorie- und Praxisphasen. Anhand der drei genannten Phänomene wurden zu Beginn Herausforderungen und Problemstellungen der Operationalisierung geisteswissenschaftlicher Konzepte reflektiert. Im Anschluss daran konnten die Teilnehmenden eigenen Interessen folgend eines der Beispiele auswählen und praktisch bearbeiten.

Am Anfang stand die manuelle Annotation: Anhand eines Textauszugs und vorbereiteter Annotationsrichtlinien annotierten sie ihr gewähltes Textphänomen und präzisierten und erweiterten dabei die Richtlinien. In einer ersten Diskussionsrunde wurden die gesammelten Erfahrungen und Ergebnisse sowie der Annotationsprozess besprochen.

Für die anschließende Erprobung der indirekten Operationalisierung stellten wir einen ,Baukasten“ aus verschiedenen Python- und R-Skripten web-basiert in einem Jupyter-Notebook ${ }^{5}$ zur Verfügung. Die Skripte waren auf das gewählte Phänomen zugeschnitten und boten den Teilnehmenden die Möglichkeit einer computergestützten Annäherung daran. Die Teilnehmenden konnten in Kleingrup-

5 https://jupyter.org/ 
pen arbeiten und dabei auf verschiedene Methoden der Datenanalyse zurückgreifen, Parameter justieren, manuelle Eigenschaften an- oder abwählen und die Ergebnisse einer maschinellen Klassifikation visualisieren. Hierbei konnten die Teilnehmenden auch das in der praktischen Annotationsrunde generierte Vorwissen einbringen. Die Verwendung von Jupyter-Notebooks erlaubte eine iterative Vorgehensweise, so dass die Teilnehmenden die benötigten Schritte einzeln und bei Bedarf erneut ausführen konnten, um die Ergebnisse zu aktualisieren. Außerdem erlaubte die Visualisierung der Ergebnisse ein direktes Feedback zur Parametrisierung, wodurch nachvollziehbar wurde, ob das gewählte Phänomen sinnvoll mit den gewählten Einstellungen zu fassen war. Eine abschließende Diskussion wertete die gesammelten Ergebnisse aus und erörterte, inwieweit sich die Anwendungsfälle angemessen modellieren ließen.

\section{Coaching}

\subsection{Ziele und Motivation}

Eine Erfahrung, die wir sowohl CRETA-intern als auch bei den Workshops gemacht haben, war, dass die Teilnehmenden oft sehr spezifische Probleme zu lösen versuchten und dass die Übertragung des Gelernten auf die konkreten Fragen, Probleme und Daten eine Herausforderung darstellte. Durch den Fokus auf einzelne methodische Bausteine konnte die Einbettung in den Gesamtzusammenhang einer vollständigen Forschungsarbeit im Rahmen eines Workshops nur lückenhaft und prototypisch behandelt werden. Daraus ergab sich der Bedarf für ein weiteres Schulungsangebot, bei dem wir auf die individuellen Herausforderungen der Forschungsarbeiten und Rahmenbedingungen für die Forschenden eingehen wollten.

Grundidee des Coachings war, mit den Gecoachten (im Folgenden, Coachees') in individuellen Gesprächsrunden einen konkreten Plan zu erarbeiten, wie sie sich ihrer textanalytischen Kernfrage nähern können. Dazu gehörte etwa die Einteilung in geeignete Teilfragen, deren konkrete Operationalisierung, die Identifikation von relevanten Ressourcen (Tools/Methoden/Korpora), sowie ggf. Publikationsstrategien für Teilergebnisse. Ziel des Coachings war also, einen konkreten Arbeitsplan zu entwickeln, auf Basis dessen die Coachees dann (weiter-)arbeiten konnten - die Arbeit am Projekt sollte also noch relativ am Anfang stehen. Zielgruppe waren dementsprechend Promovierende, die einen geisteswissenschaftlichen Hintergrund hatten und eine grundsätzlich geeignete textanalytische Frage- 
stellung verfolgten oder verfolgen wollten. ${ }^{6}$ Interessenten mussten sich mit einem Exposé zu ihrem Projekt, einem kurzen Lebenslauf sowie ggf. bereits erfolgten einschlägigen Publikationen bewerben.

Die Coaches wurden so gewählt, dass eine Bandbreite an Themen und Disziplinen von ihnen vertreten werden konnte. Neben Beteiligten in CRETA konnte auch eine externe Kooperationspartnerin gewonnen werden, mit der wir in der Vergangenheit bereits in verschiedenen Formen kooperiert hatten. Als Coaches standen bereit: Evelyn Gius (Digital Philology/Neuere Deutsche Literaturwissenschaft, TU Darmstadt), Gerhard Kremer (Computerlinguistik, Universität Stuttgart), Jonas Kuhn (Computerlinguistik, Universität Stuttgart), Janis Pagel (Computerlinguistik, Universität Stuttgart), Axel Pichler (Philosophie/Literaturwissenschaft, Universität Stuttgart), Nils Reiter (Computerlinguistik/Digital Humanities, Universität Stuttgart), Gabriel Viehhauser (Digital Humanities, Universität Stuttgart).

\subsection{Ablauf}

Das CRETA-Coaching bestand konkret aus fünf Terminen, die über einen Zeitraum von etwa zehn Tagen gestreut waren:

- Einem einwöchigen Kurs zur reflektierten Textanalyse, der auch im Rahmen der DGfS-CL-Herbstschule $2019^{7}$ angeboten wurde (,Reflected Text Analytics beyond Linguistics', Nils Reiter). Darin wurden einige methodische Grundlagen, vor allem zu Annotation, maschinellem Lernen und Evaluation, gelegt. Ausgehend von etablierten textanalytischen Verfahren aus der Computerlinguistik wurde dabei auch erarbeitet, wie diese Verfahren für andere textwissenschaftliche Fragestellungen eingesetzt werden können. Im Kurs wurde auch das oben beschriebene hackatorial eingesetzt.

- Im Fokus der zweitägigen CRETA-Werkstatt (s. o.) stand der Austausch über konkrete Arbeiten und Daten in größerer Runde. Neben Arbeiten von CRETABeteiligten konnten die Coachees ihre Projekte vorstellen und im Anschluss in themenspezifischen Arbeitsgruppen darüber diskutieren.

- In drei individuellen Coaching-Terminen trafen die Coachees mit jeweils zwei Coaches zusammen, die sich auf Basis der vorab eingereichten Exposés auch

\footnotetext{
6 Eine frühzeitige Einbeziehung der Betreuenden wurde den Promovierenden empfohlen und hat auch stattgefunden.

7 https://dgfs-clschool19.github.io
} 
Tab. 4: Zeitliche Struktur des Coachings

\begin{tabular}{llll}
\hline & Montag - Freitag & Montag & Dienstag \\
\hline Vormittag & $\begin{array}{l}\text { Zwei individuelle Coaching- } \\
\text { Termine }\end{array}$ & CRETA-Werkstatt & CRETA-Werkstatt \\
Nachmittag & $\begin{array}{l}\text { Kurs „Reflected Text Analy- } \\
\text { tics beyond Linguistics“ }\end{array}$ & CRETA-Werkstatt & Abschluss-Coaching \\
\hline
\end{tabular}

bereits mit den Projekten auseinandergesetzt hatten. Die Coaches wechselten sich ab, so dass eine Vielfalt an Perspektiven berücksichtigt werden konnte.

Tabelle 4 gibt einen Überblick über die zeitliche Struktur des Coachings. Der Abstand zwischen einzelnen Coaching-Terminen betrug einige Tage, damit a) die Coachees genug Zeit hatten, die Gespräche zu reflektieren, und b) um die Möglichkeit zu haben, kleinere Experimente oder Analysen zwischen den Terminen durchzuführen (z. B. eine Statistik über die Wortformen zu extrahieren).

Zur Vorbereitung und Strukturierung der Coachings wurden den Coaches im Vorfeld Leitfragen ausgehändigt, die im Gespräch relevant sein könnten (Abbildung 1). Die Leitfragen deckten verschiedene Aspekte sowie wiederkehrende Probleme bei DH-Forschungen ab, die je nach Forschungsfrage unterschiedlich relevant waren.

\subsection{Erfahrungen}

Aus CRETA-Perspektive war das Coaching in mehrfacher Hinsicht ertragreich. Aufgrund der unterschiedlichen disziplinären Kontexte, aus denen die Coachees stammten, sowie der Tatsache, dass sich die Coachings bereits stark an konkreten Tasks orientierten sollten bzw. diese zu entwickeln suchten, war es insbesondere notwendig, eingangs den fachspezifischen Rahmen der jeweiligen Projekte abzustecken. Dazu standen zwar erste Informationen zu den von den Coachees als relevant erachteten Methoden und Kontexten in Form der eingereichten Exposées und Lebensläufe zur Verfügung. Nicht absehbar waren jedoch die arbeitspraktischen Konsequenzen im Umgang mit diesen fachspezifischen Voraussetzungen und ihr Verhältnis zu dem in CRETA entwickelten Workflow (Pichler und Reiter 2020).

Erstens galt es daher zu klären, ob und wie sich die von den jeweiligen Projekten vorgegebenen Fragestellungen operationalisieren ließen. D. h. es war zu überprüfen, ob sich die Leitfragen in Teilfragen unterteilen ließen, die weiter in prak- 
- Task

- Was genau ist der Task?

- Ist er verwandt mit existierenden Tasks, z. B. aus der Computerlinguistik oder den Digital Humanities?

- Gibt es ein Evaluationsszenario für den Task?

- Wie generisch ist der Task? Wäre er auch auf anderen Daten/Epochen/Gattungen/Textsorten interessant?

- Existieren bereits Annotationsrichtlinien oder -schemata?

- Bezug zu CRETA

- Gibt es Anknüpfungspunkte zu CRETA?

- Berührt die Fragestellung das Forschungsgebiet eines/einer CRETA-Angehörigen?

- Gibt es Möglichkeiten für Kooperationen?

- Rahmenbedingungen

- Was sind die vorliegenden Rahmenbedingungen für das Projekt?

- Wie ist das Projekt/Zentrum/Institut zusammengesetzt, an dem das Projekt durchgeführt werden soll?

- Gibt es vor Ort Kooperationen mit der Informatik?

- Daten

- Woraus genau bestehen die Daten, wie sind sie zusammengesetzt?

- Liegen die Daten in digitaler Form vor? Wie groß ist die Datenmenge?

- Annotationen

- Wurden bereits Beispieldaten annotiert?

- Könnten testweise (z. B. zum zweiten Termin) Annotationen erzeugt werden, die dann gemeinsam diskutiert werden können?

- Wie schwer ist die Annotationsaufgabe? Wie hoch ist das inter-annotator agreement?

- Kann die Annotation von Laien oder Experten durchgeführt werden?

- Automatische Erkennung

- Welche (linguistischen) Vorverarbeitungsschritte würden bei einer Operationalisierung helfen? Welche Tools existieren dafür, sind verfügbar, für welche Sprache(n)?

- Gibt es Möglichkeiten, annotierte Daten indirekt zu generieren? Sind Fehler in diesen Daten schlimm?

- Wie könnten Visualisierungen des Ergebnisses aussehen?

- Was sind erwartete Ergebnisse, wie sieht ein mögliches Ergebnis aus?

- Verschiedenes

- Was sind mögliche Publikationsorte für (Zwischen-)Ergebnisse?

- Welche Vorkenntnisse liegen seitens der Coachees vor?

Abb. 1: Leitfragen, die den Coaches im Vorfeld des Coachings ausgehändigt wurden 
tisch umsetzbare Aufgaben auf Basis konkreter und präziser Handlungsanweisungen überführt werden konnten. Dabei war nicht nur die Operationalisierbarkeit selbst zu berücksichtigen, sondern auch zu klären, wie eine derartig in Teilaufgaben unterteilte Fragestellung mit den fachspezifischen Konventionen zu vereinbaren war. $\mathrm{Zu}$ letzteren zählen nicht nur die bereits angesprochenen Kontexte und fachspezifischen Methoden, sondern auch soziale Konventionen inklusive ihrer Auswirkungen auf die Karriereplanung. Damit diese Fragen in drei CoachingSitzungen adäquat behandelt werden konnten, war eine eingehende Auseinandersetzung mit den Projekten und der Austausch unter den Coaches im Vorfeld nötig.

Zweitens stellte das Coaching eine Möglichkeit dar, den CRETA-Workflow auf im Entstehen begriffene Forschungsprojekte anzuwenden. Die Coachings stellten somit - nach der CRETA-internen Anwendung - einen zweiten Praxistest für das besagte Verfahren dar. Dabei hat der CRETA-Workflow sowohl dazu beigetragen, die Gespräche zu strukturieren als auch die Zusammenhänge der einzelnen Arbeitsschritte $\mathrm{zu}$ verdeutlichen. Nicht zuletzt haben die im Coaching diskutierten Fragestellungen wesentlich zu einer Schärfung des in CRETA entwickelten Workflow (Pichler und Reiter 2020) beigetragen.

Wie die im folgenden partiell abgedruckten Erfahrungsberichte von zwei Teilnehmerinnen, die ursprünglich im Weblog des DHd-Verbandes ${ }^{8}$ veröffentlicht wurden, belegen, ist es im Zuge der Coachings gelungen, die beiden oben genannten Punkte zur Zufriedenheit der Teilnehmerinnen zusammenzuführen:

Das Coaching war - metaphorisch gesagt - ein Schlaraffenland für junge WissenschaftlerInnen. [...]

Die Teilnahme an der CRETA-Werkstatt stellte sich somit als krönender Abschluss heraus, bei dem wir Coachees von der komprimierten Expertise der Beteiligten profitieren konnten, mit kritischen Fragen konfrontiert wurden, aber auch konstruktives Feedback und konkrete Vorschläge zur Umsetzbarkeit unserer jeweiligen Projekte sowie Ideen für Ausweitungen erhielten und neue Forschungskontakte knüpfen konnten. (Guhr 2019)

Durch den engen Austausch mit unterschiedlichen FachvertreterInnen, die allesamt interdisziplinäres Arbeiten gewohnt sind und so gute Tipps und Einschätzungen geben konnten, entstand eine äußerst produktive und mehrdimensionale Auseinandersetzung mit der Forschungsfrage meiner Dissertation. Die Arbeit eines Promovenden - also die intensive Textarbeit, Ausarbeitung von Hypothesen und Instrumentarien -, die ja oft nur im stillen Kämmerlein stattfindet, wurde so einmal ganz offen gelegt und konstruktiv erörtert. Es entstand ein mehr als anregender Austausch, bei dem verschiedene Handlungsoptionen und Operationalisierungen durchgespielt wurden und am Ende eine Pipeline für konkrete nächs-

8 https://dhd-blog.org 
te Schritte entstand. Dabei wurden die spezifischen Fragen der Literaturwissenschaft stets im Blick behalten und mit Methodiken der Computerlinguistik flankiert. (Schmitt 2019)

Zum Erfolg trug insbesondere die Tatsache bei, dass an jedem individuellen Coachingtermin Coaches aus der Computerlinguistik und den traditionellen Geisteswissenschaften teilnahmen, die im Rahmen von CRETA bereits zusammengearbeitet hatten. Auf diese Weise war eine zentrale Grundlage für den interdisziplinären Dialog mit den Coachees gegeben, dessen Bedeutung nicht unterschätzt werden darf: ein gemeinsames Vokabular bzw. das Wissen darum, wo - je nach Disziplin - Begriffe eine abweichende Bedeutung haben. Die Beherrschung dieses interdisziplinären ,Sprachspiels‘ durch die Coaches machte die Gespräche erst konstruktiv.

\section{Fazit}

Obwohl Disseminationsarbeit in vielen Fällen zunächst als zusätzlicher Aufwand empfunden wird und daher Gefahr läuft, hinter den ,wirklich wichtigen' Dingen (z. B. der Arbeit an der eigenen Dissertation) zurückzutreten, haben wir mit den hier dargestellten Aktivitäten positive Erfahrungen gemacht: Bedacht gewählte Disseminationsvorhaben entfalten nicht nur eine Wahrnehmbarkeit nach außen, indem sie anderen ermöglichen, an den eigenen Projektergebnissen teilzuhaben und auf sie aufzubauen. Dies umfasst insbesondere auch methodische, pragmatische und organisatorische Erkenntnisse und Erfahrungen, die in wissenschaftlichen Publikationen nur schlecht oder gar nicht Platz finden.

Auch intern können diese Aktivitäten eine positive Wirkung entfalten. $\mathrm{Zu}$ nächst dienen sie dem Bündeln und zielgerichteten Ausrichten der internen Arbeit. Dadurch, dass relativ klare Anforderungen innerhalb eines definierten Zeitgerüstes herrschen, fokussieren sich die Beteiligten in anderer Form auf die anstehenden Arbeiten: Die Aufbereitung von intern erarbeiteten Inhalten für Workshops und Lehre zwingt zur Präzision und zur Konzentration auf das Wesentliche, wovon alle Beteiligten profitieren. Die Vorbereitung eines Workshops verpflichtet dazu, sehr genau über den Workshop zu reden.

Weiterhin sind derartige Aktivitäten eine Gelegenheit für das Projektteam, die unterschiedlichen fachlichen Perspektiven auf das Gebiet der Digital Humanities kennenzulernen und trotz ungewohnter Denkweisen die Gemeinsamkeiten zu finden, die das Team verbinden und die Zusammenarbeit stärken. Das begünstigt insgesamt die Aufgeschlossenheit aller Beteiligten gegenüber ungewohnten Ansätzen oder Methoden. 
Auch die Aktivitäten an sich und der Austausch mit den Teilnehmenden (von Workshops bzw. Coaching) haben die Arbeiten in CRETA vorangebracht. Dadurch, dass die Teilnehmenden versuchen, die erlernten Methoden auf ihre eigenen Fragestellungen anzuwenden, werden diese Methoden auch mit Situationen konfrontiert, die innerhalb des Zentrums nicht vorkommen (etwa weil niemand mit einer bestimmten Art Daten arbeitet). Selbst wenn das Ergebnis dieser Konfrontation dann mitunter ist, dass bestimmte Methoden auf bestimmte Daten nicht sinnvoll anwendbar sind, ist dies ein methodischer Fortschritt, der ohne die Konfrontation nicht erfolgt wäre. Insofern stellt selbst dieser Fall noch einen Erkenntnisgewinn dar.

Die Erfahrungen in CRETA lehren dementsprechend, dass es ein interdisziplinäres Forschungsvorhaben bedeutend voranbringen kann, frühzeitig über den Tellerrand des eigenen Projektes zu blicken und nach außen gerichtete Aktivitäten durchzuführen. Gerade im vergleichsweise jungen Bereich der Digital Humanities, in dem noch dazu ein bisweilen unübersichtlicher Methodenimport aus verschiedensten Disziplinen stattfindet, sind diese Angebote nicht nur beliebt und hoch frequentiert, sondern wirken sich auch innerhalb des eigenen Projekts positiv aus.

\section{Literatur}

Barth, Florian (2020). „Annotation narrativer Ebenen und narrativer Akte“. In: Reflektierte Algorithmische Textanalyse. Hrsg. von Nils Reiter, Axel Pichler und Jonas Kuhn. Berlin: De Gruyter, S. 423-438.

Gius, Evelyn (2019). „Computationelle Textanalysen als fünfdimensionales Problem: Ein Modell zur Beschreibung von Komplexität“. Pamphlet 8. Digital Humanities Cooperation. URL: https://www.digitalhumanitiescooperation.de/wp-content/uploads/2019/12/pamphlet_ gius_2.0.pdf (besucht am 1. Juni 2020).

Gius, Evelyn und Janina Jacke (2017). „The Hermeneutic Profit of Annotation: On Preventing and Fostering Disagreement in Literary Analysis“. In: International Journal of Humanities and Arts Computing 11.2, S. 233-254.

Guhr, Svenja (2019). Erfahrungsbericht über ein Schlaraffenland für NachwuchswissenschaftlerInnen. URL: https://dhd-blog.org/?p=12265 (besucht am 1. Juni 2020).

Jacke, Janina (2014). „Is There a Context-Free Way of Understanding Texts? The Case of Structuralist Narratology“. In: Journal of Literary Theory 8, S. 118-139.

Jannidis, Fotis, Isabella Reger, Markus Krug, Lukas Weimer, Luisa Macharowsky und Frank Puppe (2016). „Comparison of Methods for the Identification of Main Characters in German Novels“. In: Digital Humanities 2016: Conference Abstracts. Kraków, Poland: Jagiellonian University \& Pedagogical University, S. 578-582.

Ketschik, Nora, André Blessing, Sandra Murr, Maximilian Overbeck und Axel Pichler (2020). „Interdisziplinäre Annotation von Entitätenreferenzen“. In: Reflektierte Algorithmische 
Textanalyse. Hrsg. von Nils Reiter, Axel Pichler und Jonas Kuhn. Berlin: De Gruyter, S. 204236.

Ketschik, Nora, Sandra Murr, Benjamin Krautter und Yvonne Zimmermann (2020). „Zur Theorie von Erzählebenen und ihrer Annotation im digitalen Kontext“. In: Reflektierte Algorithmische Textanalyse. Hrsg. von Nils Reiter, Axel Pichler und Jonas Kuhn. Berlin: De Gruyter, S. 440-464.

Lengwiler, Martin (2005). „Erfolgreich Inter-Disziplinieren“. In: WZB-Mitteilungen 107, S. 52-55. Moretti, Franco (2013). „,Operationalizing': or, the Function of Measurement in Modern Literary Theory“. Pamphlet 6. Stanford Literary Lab, S. 1-13. URL: https: / / litlab. stanford. edu / LiteraryLabPamphlet6.pdf (besucht am 1. Juni 2020).

Pagel, Janis, Nils Reiter, Ina Rösiger und Sarah Schulz (2020). „Annotation als flexibel einsetzbare Methode“. In: Reflektierte Algorithmische Textanalyse. Hrsg. von Nils Reiter, Axel Pichler und Jonas Kuhn. Berlin: De Gruyter, S. 125-141.

Pichler, Axel und Nils Reiter (2020). „Reflektierte Textanalyse“. In: Reflektierte Algorithmische Textanalyse. Hrsg. von Nils Reiter, Axel Pichler und Jonas Kuhn. Berlin: De Gruyter, S. 4359.

Reiter, Nils, Jonas Kuhn und Marcus Willand (2017). „To GUI or not to GUI?“ In: INFORMATIK 2017. Bd. 275. Lecture Notes in Informatics (LNI). Chemnitz, Germany: Gesellschaft für Informatik e. V., S. 1179-1184. DoI: 10.18420/in2017_119.

Reiter, Nils und Marcus Willand (2018). „Poetologischer Anspruch und dramatische Wirklichkeit: Indirekte Operationalisierung in der digitalen Dramenanalyse Shakespeares natürliche Figuren im deutschen Drama des 18. Jahrhunderts“. In: Quantitative Ansätze in den Literaturund Geisteswissenschaften. Systematische und historische Perspektiven. Hrsg. von Toni Bernhart, Marcus Willand, Andrea Albrecht und Sandra Richter. Berlin: De Gruyter, S. 4576. DoI: 10.1515/9783110523300-003.

Richter, Sandra (2020). „Reading with the workflow: Arbeitsprozesse in den Computational Literary Studies - Beiträge zur Empirisierung literaturwissenschaftlicher Verfahren“. In: Reflektierte Algorithmische Textanalyse. Hrsg. von Nils Reiter, Axel Pichler und Jonas Kuhn. Berlin: De Gruyter, S. 143-168.

Sack, Graham Alexander (2011). „Simulating Plot: Towards a Generative Model of Narrative Structure“. In: AAAI Symposium, S. 127-136.

Schmitt, Anna (2019). Das Creta-Coaching an der Universität Stuttgart. URL: https : / / dhd blog.org/?p=12551 (besucht am 1. Juni 2020).

Suditsch, Isabel (2017). „Interdisziplinärer Fachsprachenkontakt - Fallstudie Arena2036“. Diss. Stuttgart University.

Willand, Marcus, Evelyn Gius und Nils Reiter (2020). „SANTA: Idee und Durchführung“. In: Reflektierte Algorithmische Textanalyse. Hrsg. von Nils Reiter, Axel Pichler und Jonas Kuhn. Berlin: De Gruyter, S. 391-422. 